\title{
Fermion resonances in quiver theories with a pNGB Higgs
}

\author{
Gustavo Burdman, Pedro Ormonde and Victor Peralta \\ Instituto de Física, Universidade de São Paulo, \\ R. do Matão 187, São Paulo, SP 05508-900, Brazil \\ E-mail: burdman@if.usp.br, pedroh_ormonde@hotmail.com, \\ victorpc@if.usp.br
}

ABSTRACT: Hierarchical quiver models can be used to build theories of electroweak symmetry breaking and natural models of flavor with a pseudo-Nambu-Goldstone boson (pNGB) Higgs. They are cousins of similar models in extra-dimensional theories in anti-de Sitter backgrounds, and can be obtained from them by coarse deconstruction. We consider the fermion excitations in these models, focusing on the quark sector and studying its generic features and phenomenology. We show that, unlike in the continuum case, the spectrum is strongly flavor dependent. To study the phenomenology of the quark excitations we compute their couplings to the Higgs sector and the gauge excitations which determine both their single-production and their decays. We show how the generic features of quiver theories with a pNGB Higgs translate, through the spectrum of quark excitations and their couplings, into a distinct phenomenology at the LHC.

Keywords: Beyond Standard Model, Higgs Physics, Technicolor and Composite Models

ARXIV EPRINT: 1408.1320 


\section{Contents}

1 Introduction 1

2 Quiver theories of EWSB with a pNGB Higgs 3

$\begin{array}{lll}3 & \text { The Fermion resonances in quiver models } & 6\end{array}$

4 Couplings of the excited fermions 11

$\begin{array}{llr}5 & \text { Phenomenology } & 14\end{array}$

6 Conclusions and outlook $\quad 17$

\section{Introduction}

The recent discovery of the Higgs boson $[1,2]$ completes the necessary spectrum of the standard model (SM). Barring small deviations in the Higgs couplings, the SM can describe all the data available to date. The agreement with experiment of such a renormalizable theory, including the renormalizable Higgs potential, suggests that the SM is valid up to energies well above the weak scale. On the other hand, for the Higgs mass $m_{h}$ to be well below the SM cutoff $\Lambda_{\mathrm{SM}}$, whatever this might be, a large tuning of the order of one part in $m_{h}^{2} / \Lambda_{\mathrm{SM}}^{2}$ is necessary, resulting in the hierarchy problem. This tuning is always present unless there is a symmetry broken just above the weak scale that reduces the quadratic sensitivity of the ultraviolet boundary conditions. The two options that are still compatible with data are supersymmetric extensions of the SM [3], and the possibility that the Higgs is a pNGB [4]. In the latter case, which we consider here, it is assumed that the Higgs is part of a NGB from the spontaneous breaking of a large global symmetry. Explicit breaking, typically induced by the SM interactions, result in a Higgs potential. To this class belong Little Higgs models [5-7], as well as the so-called composite Higgs models [8-10], which can be thought of as related by holography to five-dimensional theories in anti-de Sitter backgrounds $\left(\mathrm{AdS}_{5}\right)$ [11]. In general, composite Higgs models (CHM) with a pNGB Higgs are associated with a strong sector and result in strongly coupled resonances. This puts important constraints on the models, both through electroweak precision [12-14] as well as flavor bounds $[13,15]$.

On the other hand, it is possible to build pNGB Higgs models in four-dimensional (4D) field theories that have very similar features. These theories, which we call quiver theories from now on, can be obtained from the coarse deconstruction $[16,17]$ of the $\mathrm{AdS}_{5}$ models $[18,19]$. They have qualitative similarities but some crucial quantitative differences with their five-dimensional (5D) counterparts. In particular, the resulting $4 \mathrm{D}$ theories are weakly coupled, and as a consequence will present less problems with indirect bounds, as 
it was shown in ref. [20]. In general, it is possible to obtain any coarsely deconstructed, weakly coupled version of any CHM.

The complete $4 \mathrm{D}$ theory from $\mathrm{AdS}_{5}$ deconstruction including fermions was first presented in ref. [21]. The idea of using these quiver theories to study models of electroweak symmetry breaking and fermion masses was further advanced in ref. [20], where the flavor bounds of full solutions to the quark masses and mixing matrix where obtained. Ref. [22] started exploring the phenomenology of these theories at the LHC by considering the minimal spectrum of gauge boson excitations. Although the bounds obtained in [22] depend somewhat on the number of sites in the quiver theory, they are typically around $2.5 \mathrm{TeV}$ for the gluon excitation (assuming $\mathrm{SU}(3)_{c}$ "propagates" in the quiver), and about $(1.7-2.0) \mathrm{TeV}$ for the photon and $Z$ excitations. The latter bound is inescapable since the electroweak gauge boson excitations must be present in any realization of the model, whereas it is possible to consider quiver theories of electroweak symmetry breaking (EWSB) without having gluon excitations.

In this paper, we will consider the fermion excitations in quiver theories where the Higgs is a pNGB. The details of the pNGB Higgs sector are studied elsewhere [23]. Here it will suffice to consider the minimum number of elements of the pNGB scenario in quiver theories that will allow us to compute or estimate the fermion excitation couplings to the Higgs sector, which will be important for both their production and decays. In this spirit, we will not define the gauge groups propagating in the quiver (unless for illustration purposes). This implies that we will only consider the fermion excitations corresponding to SM zero modes, and will ignore other excitations that will depend on the fermion representations on the quiver theory.

In order to compute the fermion excitation spectrum we will take flavor solutions from ref. [20]. These solutions, consistent with quark flavor physics, determine the localization of zero-mode fermions in the quiver diagram. As we will see below, the fermion excitation spectrum has a rather distinct dependence on the localization parameters, very different from the one in the continuum theories. The aim of the paper is to obtain all the relevant information, i.e. spectrum and couplings, that will allow us to study the phenomenology of these fermion excitations. Here we concentrate on the quark excitations, for which the zeromode solutions were obtained in [20]. The lepton excitations will be studied separately [24].

Other works have considered similar 4D constructions. For instance in refs. [25] and [26] two-site models were considered to capture the essence of composite models. In ref. [27] a three-site Higgsless model is studied. The three-site model with a pNGB Higgs of ref. [28] is closest to our set up, although with a particular choice of group. Our aim is to generalize the study of quiver theories for various values of the number of sites $N$ and find their generic features independently of the details of the model.

The rest of the paper is organized as follows: in section 2 we present the general features of quiver theories of EWSB with a pNGB Higgs. This will set up the model in which the excited fermions will be studied. In section 3 we focus on the spectrum of fermion resonances, as well as on the wave-functions of the zero-mode and the excited states. These will be used to compute the couplings of the excited fermions to various states, in particular excited gauge bosons and the Higgs sector in section 4, since these couplings will determine the phenomenology of these resonances. These couplings are then 
used to begin the study of the phenomenology of these excited fermions in section 5 . We finally conclude in section 6 .

\section{Quiver theories of EWSB with a pNGB Higgs}

The general construction of the 4 D theory starts with a product gauge group $G_{0} \times G_{1} \times$ $\cdots G_{j} \times G_{j+1} \cdots G_{N}$. In addition, we have a set of scalar link fields $\Phi_{j}$, with $j=1$ to $N$, transforming as bi-fundamentals under $G_{j-1} \times G_{j}$. The action for the theory is

$$
S=\int d^{4} x\left\{-\sum_{j=0}^{N} \frac{1}{2} \operatorname{Tr}\left[F_{\mu \nu}^{(j)} F^{\mu \nu(j)}\right]+\sum_{j=1}^{N} \operatorname{Tr}\left[\left(D_{\mu} \Phi_{j}\right)^{\dagger} D^{\mu} \Phi_{j}\right]-V\left(\Phi_{j}\right)+\ldots\right\}
$$

where the traces are over the groups' generators, and the dots at the end correspond to terms involving fermions and will be discussed in the next section. We assume that the potentials for the link fields give them a vacuum expectation value (VEV) which breaks $G_{j-1} \times G_{j}$ down to the diagonal group, resulting in non-linear sigma models for the $\Phi$ 's

$$
\Phi_{j}=\frac{v_{j}}{\sqrt{2}} e^{i \sqrt{2} \pi_{j}^{a} t^{a} / v_{j}}
$$

where the $t^{a}$ 's are the broken generators, the $\pi_{j}^{a}$ the Nambu-Goldstone Bosons (NGB); and $v_{j}$ are the VEVs of the link fields. We consider here the situation where the VEVs are ordered in such a way that $v_{1} \cdots>v_{j} \cdots>v_{N}$. This choice is motivated by the goal of creating a large hierarchy of scales between the high energy VEVs and the infra-red (IR) ones close to the gauge group $N$. As we see below, this also makes contact with the $\mathrm{AdS}_{5}$ setup in the continuum limit.

The idea behind the model with the action in (2.1) is similar to the one in ref. [4]. We imagine that the link fields arise as a consequence of fermion condensation at the approprate scale. For instance the link field $\Phi_{j}$ is a consequence of the condensation of fermions transforming in the fundamental of $G_{j-1}$ and anti-fundamental of $G_{j}$ at the scale $\sim v_{j}$. So the effective theory in (2.1) is the result of these strong gauge groups having all these various infrared scales. The possible mixings between neighboring link fields, e.g. $\Phi_{j-1}^{2} \Phi_{j}^{2}$, are induced at one loop order by gauge boson loops, and will not affect the stability of the VEVs in the quiver which is assumed to be determined by the underlying strong dynamics. The potentials in (2.1) reflect interactions of the heavier states (e.g. $\sigma_{j}$ 's) and are not relevant for the physics of the gauge excitations and the pNGBs.

We parametrize the ordering by defining the VEVs as

$$
v_{j} \equiv v q^{j}
$$

where $0<q<1$ is a dimensionless constant, and $v$ is a UV mass scale that can be regarded as the UV cutoff. In this particular example we assume that the all the gauge groups are identical. This will not always be the case, as we will se below. The gauge couplings satisfy

$$
g_{0}(v)=g_{1}\left(v_{1}\right)=\cdots=g_{j}\left(v_{j}\right)=g_{j+1}\left(v_{j+1}\right)=\cdots \equiv g .
$$

The model can be illustrated by the quiver diagram of figure 1 . 


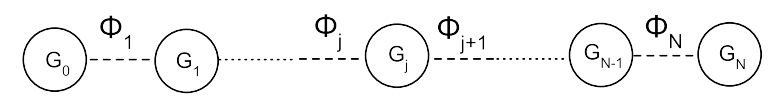

Figure 1. Quiver diagram for the theory described by (2.1).

This purely $4 \mathrm{D}$ theory can be obtained from deconstructing an extra-dimensional theory in an $\mathrm{AdS}_{5}$ background $[18,19,29,30]$. Discretizing a $5 \mathrm{D}$ gauge theory in an $\mathrm{AdS}_{5}$ background by a discrete interval $1 / g v$ in $N$ intervals results in the action (2.1), with the appropriate identification of the 5D gauge coupling, plus the matching

$$
q \leftrightarrow e^{-k / g v}
$$

However, in order for the $4 \mathrm{D}$ theory defined by (2.1) to remain a good description of the continuum 5D theory, the $\mathrm{AdS}_{5}$ curvature should satisfy $k<v$, or $q$ close to 1 . When this is satisfied, getting closer to the continuum limit by increasing the number of sites $N$ guarantees an increasing similarity with the 5D theory [30]. For instance, generating the hierarchy between the Planck and the weak scales while satisfying $k<v$ requires typically that $N>35$, which results in a low energy theory very close to the continuum one. Under these conditions, $4 \mathrm{D}$ theories with $k<v$ are just discrete descriptions of the $\mathrm{AdS}_{5}$ theory.

On the other hand, if we consider (2.1) as just a $4 \mathrm{D}$ theory, we are free to make use of values of $q$ far from what would constitute the continuum $5 \mathrm{D}$ limit, i.e. $q \ll 1$. In these theories it will be possible to obtain a large hierarchy of scales with smaller values of $N$, as low as just a few. For instance, if $v \lesssim M_{P}$ and $v_{N} \simeq O(1) \mathrm{TeV}$, then we can write

$$
q=10^{-16 / N}
$$

For instance, for $N=4$ we have $q=10^{-4}$, very far from the continuum limit. The theories resulting in these region of the parameters of the action in (2.1) will have a very different behavior than a mere discretization of $\mathrm{AdS}_{5}$. Their spectrum and its properties, such as couplings to SM matter, differ significantly and therefore they merit a detailed study.

In ref. [22] we studied the generic features of the vector resonant sector of quiver theories. In order to be as model-independent as possible we studied a minimal extension of the gauge sector of the SM that would be consistent with having a pNGB Higgs, resulting in a minimum spectrum of vector resonances, for which we obtained bounds and made predictions for the LHC. In this paper, we study the fermionic resonances in these models. Just as in the case of the vector resonances, the spectrum of fermion resonances and the details of their couplings will be model dependent, i.e. it will somewhat depend on the choice of gauge symmetries propagating in the quiver diagrams. Once again, we will simplify as much as possible in order to obtain a minimal spectrum of resonances with couplings that have the correct features as imposed by the following requirements: i) the Higgs is a pNGB; ii) the hierarchy of fermion masses is obtained by fermion "localization" in the quiver diagram, as shown in ref. [20].

The first requirement implies that the Higgs is extracted from the link fields in the quiver, and therefore propagates through it in a very specific way [23]. In fact, the Higgs 
must be extracted from the $\pi_{j}^{a}$ 's in the link fields in Equation (2.2). In order to achieve this, the part of the groups $G_{0}$ and $G_{N}$ in figure 1 that is gauged must be smaller than in the rest of the quiver. Specifically, only the subgroups $H_{0}$ and $H_{N}$ are gauged at these sites. The fact that the quiver gauge group is smaller, although the number of link fields remained the same, results in some NGBs remaining in the physical spectrum. These are the NGBs that cannot be removed by $H_{0}$ or $H_{N}$ gauge transformations, and therefore they transform in the cosets $G_{0} / H_{0}$ and $G_{N} / H_{N}$. A similar procedure is followed in extra-dimensional theories in order to extract the Higgs from the extra component of the gauge fields in $5 \mathrm{D}[8,9]$. To make clearer how to extract the pNGB Higgs from the link fields in quiver theories, we start by re-writing the action in (2.1) with the addition of the gauge fixing term

$$
\mathcal{L}_{\mathrm{GF}}=-\frac{1}{2 \xi} \sum_{j=0}^{N}\left[\partial^{\mu} A_{\mu}^{a j}+\xi g\left(v_{j} \pi_{j}^{a}-v_{j+1} \pi_{j+1}^{a}\right)\right]^{2}
$$

where we have considered the same gauge parameter $\xi$ for all sites for simplicity. This choice cancels all the cross terms mixing the NGBs with the gauge bosons in (2.1) that are made apparent by expanding the $\Phi_{j}$ in (2.2) in terms of the NGBs $\pi_{j}^{a}$. Doing so results in

$$
\begin{aligned}
S= & \int d^{4} x\left\{-\sum_{j=0}^{N}\left(\frac{1}{2} \operatorname{Tr}\left[F_{\mu \nu}^{(j)} F^{\mu \nu(j)}\right]-\frac{1}{2 \xi}\left(\partial^{\mu} A_{\mu}^{a j}\right)^{2}\right)+\frac{1}{2} \sum_{j=1}^{N}\left(\partial_{\mu} \pi_{j}^{a}\right)\left(\partial^{\mu} \pi_{j}^{a}\right)\right. \\
& \left.\sum_{j=1}^{N} \frac{g v_{j}^{2}}{2}\left(A_{\mu}^{a(j-1)}-A_{\mu}^{a j}\right)^{2}-\frac{1}{2} g^{2} \xi \sum_{j=0}^{N}\left(v_{j} \pi_{j}^{a}-v_{j+1} \pi_{j+1}^{a}\right)^{2}+\ldots,\right\}
\end{aligned}
$$

where the dots denote interaction terms not quadratic in the fields. The last term in (2.8) is the NGB mass matrix, which is clearly gauge dependent. In fact, making use of (2.3), it can be shown that it does not have a zero mode, and that the NGB masses are always proportional to $\sqrt{\xi}$. Thus, in the unitary gauge $\xi \rightarrow \infty$ and all NGBs decouple leaving only the longitudinal components of the massive gauge boson tower as degrees of freedom.

On the other hand, if we want to extract the Higgs from the NGBs, we need to reduce the gauge groups at sites $j=0$ and $j=N$. The NGB mass matrix in (2.8) now reads

$$
\pi^{a T} M_{\pi}^{2} \pi^{a} \equiv g^{2} \xi \sum_{j=1}^{N-1}\left(v_{j} \pi_{j}^{a}-v_{j+1} \pi_{j+1}^{a}\right)^{2},
$$

where we defined $\pi^{a} \equiv\left(\pi_{1}^{a}, \pi_{2}^{a} \ldots \pi_{N}^{a}\right)^{T}$. This mass matrix differs from the last term in (2.8) only by the limits of the sum, which result from the absence of the mixing terms between $\pi_{1}^{a}$ and $\pi_{N}^{a}$, which do not have gauge bosons to mix with due to the reduced gauge groups at sites $j=0$ and $j=N$. The matrix $M_{\pi}^{2}$ in (2.9) has null determinant, signaling the presence of a zero mode, the physical NGB. In order to extract the Higgs doublet from this NGB we must carefully choose $H_{0}$ and $H_{N}$. For instance, if the quiver groups are $\mathrm{SU}(3)_{j}$ for $0<j<N$ and we choose $H_{0}=\mathrm{SU}(2) \times \mathrm{U}(1)=H_{N}$, the zero-mass NGB will contain 
the Higgs doublet and its complex conjugate as in

$$
\pi_{j}^{a} t^{a}=\left(\begin{array}{ccc}
0 & 0 & h_{1} \\
0 & 0 & h_{2} \\
h_{1}^{*} & h_{2}^{*} & 0
\end{array}\right)
$$

where the Higgs doublet is $H=\left(h_{1} h_{2}\right)^{T}$. In general, we want to identify the combination of NGBs $\pi_{j}^{a}$ that cannot be removed by gauge transformations. In other words, what is the linear combination of the $\pi_{j}^{a}$ 's that makes up the physical NGB ? In order to do this, we look for the eigenstate for the zero mode equation:

$$
M_{\pi}^{2}\left(b_{1} \pi_{j}^{a} t^{a}, b_{2} \pi_{j}^{a} t^{a}, \ldots, b_{N} \pi_{N}^{a} t^{a}\right)^{T}=0,
$$

where the $b_{j}$ 's represent the "wave-function" of the physical NGB in the quiver. From (2.11) we see that they satisfy

$$
b_{j}=q b_{j+1}
$$

which, since $q<1$, means that the NGB wave-function is always localized towards the sites with larger values of $j$ in the quiver, or IR-localized. The physical NGB then can be expressed as

$$
\mathcal{H}=\sum_{j=1}^{N} b_{j} \pi_{j}^{a} t^{a}
$$

with the $b_{j}$ 's satisfying the normalization condition $\sum_{j=1}^{N}\left|b_{j}\right|^{2}=1$, which together with (2.12) result in

$$
b_{j}=\frac{q^{N-j}}{\sqrt{\sum_{j=1}^{N} q^{2(N-j)}}} .
$$

In practice, for the coarse deconstruction models studied here, since $q \ll 1$ the physical NGB will be highly localized very close to the site N. Thus, just as in $\operatorname{AdS}_{5}$ composite Higgs models where the Higgs is localized near the IR brane, here the Higgs is localized towards the IR site $N$. This feature is generic in that it does not depend on the details of the model, i.e. is independent of the choice of gauge groups propagating in the quiver diagram. It will be then possible to extract a lot of information regarding the couplings of the Higgs to gauge bosons and zero-mode and excited fermions without specifying the model. In the next section we introduce fermions in the quiver and obtain some of the properties of the fermion excitations. As mentioned earlier, we focus in the minimal set of fermions that have to be present regardless of the gauge groups in the quiver.

\section{The Fermion resonances in quiver models}

We consider vector-like fermions $\psi^{j}$ transforming in the fundamental representation of the groups $G_{j}$. The action of (2.1) is then enlarged by the fermion action given by

$$
S_{f}=\int d^{4} x \sum_{j=0}^{N}\left\{\bar{\psi}_{L}^{j} i \not D_{j} \psi_{L}^{j}+\bar{\psi}_{R}^{j} i \not D_{j} \psi_{R}^{j}-\left(\mu_{j} \bar{\psi}_{L}^{j} \psi_{R}^{j}+\lambda_{j} \bar{\psi}_{R}^{j-1} \Phi_{j} \psi_{L}^{j}+\text { h.c. }\right),\right\}
$$




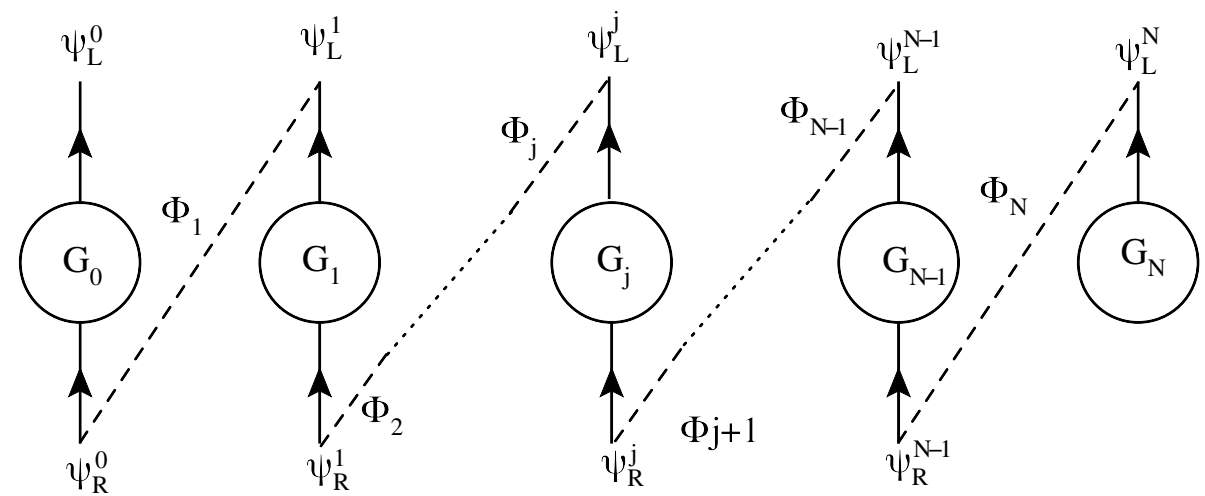

Figure 2. Quiver diagram for the theory described by (3.1), for a spectrum with a left-handed zero mode.

which is represented by the quiver diagram of figure 2 . The vector-like masses $\mu_{j}$ preserve the gauge symmetries. The Yukawa term is invariant since the links transform as $\Phi_{j} \rightarrow$ $g_{j-1} \Phi_{j} g_{j}^{\dagger}$. The Yukawa couplings are allowed to be site-dependent, which is the most general situation in the $4 \mathrm{D}$ theory. If one wanted to match to the continuum limit of the $\mathrm{AdS}_{5}$ theory we should take them to be universal, as shown in ref. [21]. In the unitary gauge we make the replacement $\Phi_{j} \rightarrow v_{j} / \sqrt{2}$, which leads to a non-diagonal mass matrix for the fermions. We diagonalize to the mass eigenstate basis through the unitary transformations

$$
\psi_{L, R}^{j}=\sum_{n=0}^{N} h_{L, R}^{j, n} \chi_{L, R}^{(n)},
$$

where the $\chi_{L, R}^{(n)}$ are the mass eigenstates. Imposing the equations of motion, results in the elements of the rotation matrices satisfying the equations [21]

$$
\begin{array}{r}
\left(\mu_{j}^{2}+\frac{\lambda_{j}^{2} v_{j}^{2}}{2}-m_{n}^{2}\right) h_{L}^{j, n}-\frac{\lambda_{j} v_{j}}{\sqrt{2}} \mu_{j-1} h_{L}^{j-1, n}-\frac{\lambda_{j+1} v_{j+1}}{\sqrt{2}} \mu_{j} h_{L}^{j+1, n}=0 \\
\left(\mu_{j}^{2}+\frac{\lambda_{j+1}^{2} v_{j+1}^{2}}{2}-m_{n}^{2}\right) h_{R}^{j, n}-\frac{\lambda_{j} v_{j}}{\sqrt{2}} \mu_{j} h_{R}^{j-1, n}-\frac{\lambda_{j+1} v_{j+1}}{\sqrt{2}} \mu_{j+1} h_{R}^{j+1, n}=0
\end{array}
$$

where $m_{n}$ is the mass of the mass eigenstate $\chi_{L, R}^{(n)}$. In order to obtain chiral zero modes, appropriate boundary conditions must be chosen. To obtain a left-handed zero mode, we must choose $h_{R}^{N, n}=0$ for all $n$, i.e. the right-handed component of the fermion at the last site must be removed. This is illustrated in figure 2. On the other hand, in order to have a right-handed zero mode, we must choose that $h_{L}^{0, n}=0$, i.e the left-handed fermion must be removed from the first site [21].

The solutions of these equations can be obtained [30] and in the continuum limit would match to the solutions for the wave-functions of the Kaluza-Klein fermions in the $\mathrm{AdS}_{5}$ [21]. But here we stay far from the continuum.

The fermion zero-modes satisfy the simple equations of motion

$$
\mu_{j} h_{L}^{j, 0}+\frac{\lambda_{j+1}}{\sqrt{2}} v_{j+1} h_{L}^{j+1,0}=0
$$


for the left-handed zero mode, and

$$
\mu_{j} h_{R}^{j, 0}+\frac{\lambda_{j}}{\sqrt{2}} v_{j} h_{R}^{j-1,0}=0
$$

for the right-handed zero mode.

We can define the localization parameter $c_{L}$ for the left-handed zero mode by [21]

$$
\sqrt{2} \frac{\mu_{j}}{v \lambda_{j+1}} \equiv-q^{j+1 / 2+c_{L}},
$$

and then consistently identify the localization parameter $c_{R}$ for a right-handed zero mode by

$$
\sqrt{2} \frac{\mu_{j}}{v \lambda_{j}}=-q^{j+1 / 2+c_{R}}
$$

Then, we can see that

$$
\frac{h_{L}^{j+1,0}}{h_{L}^{j, 0}}=q^{c_{L}-1 / 2}, \quad \frac{h_{R}^{j, 0}}{h_{R}^{j-1,0}}=q^{-\left(c_{R}+1 / 2\right)} .
$$

Thus, we have traded the ratio of vector masses to Yukawa couplings for a parameter $\left(c_{L}\right.$ or $c_{R}$ ) that will determine the fraction each fermion in the quiver diagram the zero-mode fermion contains. This particular choice is motivated in order to match the zero-mode localization in the continuum [31]. As we will see below, this means that the choice of these parameters determines the zero-mode localization. For instance, for $c_{L}>1 / 2$, the zero-mode will be UV localized (towards the "0" site), whereas this happens for the right-handed zero-mode for $c_{R}<-1 / 2$. We can now write

$$
h_{L, R}^{j, 0}=z_{L, R}^{j} h_{L, R}^{0,0},
$$

where we have defined

$$
z_{L} \equiv q^{c_{L}-1 / 2}, \quad z_{R} \equiv q^{-\left(c_{R}+1 / 2\right)} .
$$

On the other hand, the normalization conditions require that

$$
\sum_{j=0}^{N}\left|h_{L, R}^{j, 0}\right|^{2}=1
$$

which we use to obtain

$$
h_{L, R}^{0,0}=\sqrt{\frac{1-z_{L, R}^{2}}{1-z_{L, R}^{2(N+1)}}},
$$

The zero-mode wave functions are determined by the choice of the localization parameters $c_{L, R}$. These are chosen in order such that the zero-mode spectrum matches the SM spectrum. In ref. [20], solutions to the quark spectrum and the CKM matrix were found for these parameters, using the approximation of a Higgs localized in the Nth site of the quiver. As mentioned in the previous section, the pNGB Higgs wave function for small number of sites is very well approximated by N-localization. For the purpose of 

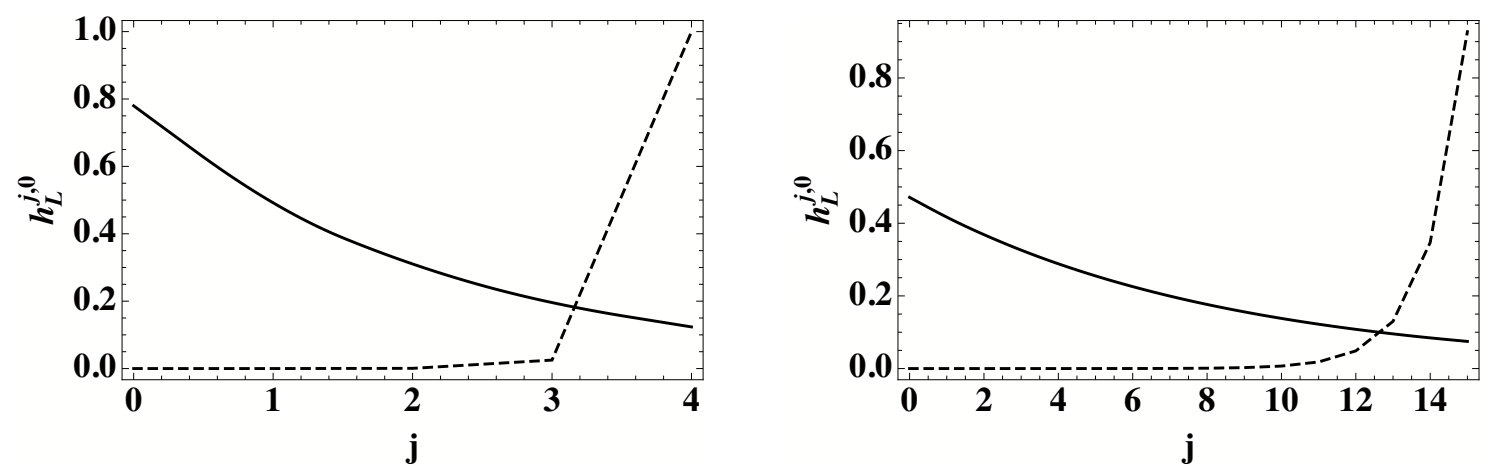

Figure 3. The wave-function of left-handed zero modes as a function of the site number $j$. Left panel: $N=4$, for $c_{L}=0.55$ (solid), $c_{L}=0.1$ (dashed). Right panel: $N=15$, for $c_{L}=0.55$ (solid), $c_{L}=0.1$ (dashed).
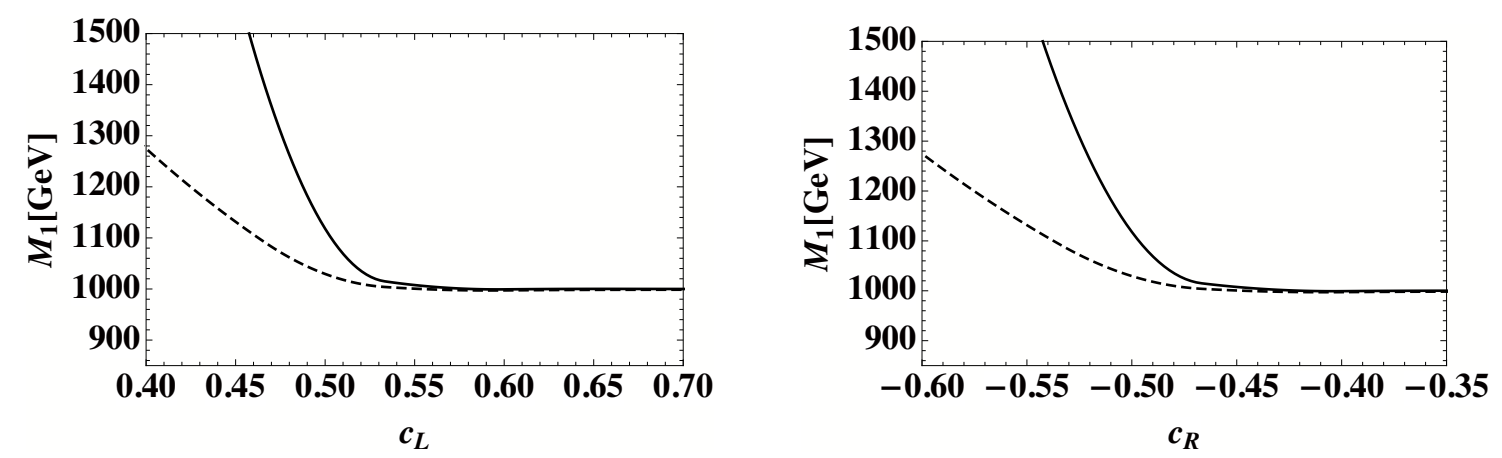

Figure 4. Left panel: the mass of the first excited fermion state in a tower with a left-handed zero mode, as a function of the localization parameter $c_{L}$, for $N=4$ (solid) and $N=15$ (dashed). Right panel: the mass of the first excited fermion state in a tower with a right-handed zero mode, as a function of the localization parameter $c_{R}$, for $N=4$ (solid) and $N=15$ (dashed).In both cases, the mass of the gauge excitation is set to $1 \mathrm{TeV}$.

the determination of the localization parameters for each fermion tower, we will use the solutions found in ref. [20]. We have checked that the use of this approximation in this case makes no significant numerical difference. For illustration, we plot the resulting zero-mode wave functions for some typical cases in figure 3, as a function of the position in the quiver diagram, $j$. In the left panel we see the wave function of a zero-mode left-handed quark with $c_{L}=0.55$ (solid line), which corresponds to ultra-violet (UV) localization, while the dashed line for $c_{L}=0.1$, corresponds to infra-red (IR) localization. For the right-handed zero modes, we can obtain analogous figures. For instance, for $c_{R}=-0.55$ and $c_{R}=-0.1$ we would obtain the same two lines of figure 3 .

For the excited fermions, we make use of the of the equations of motion in (3.3) and (3.4). Their spectrum can be obtained diagonalizing the mass matrix. As an illustration, we plot the mass of the first fermion excitation in figure 4 as a function of the localization parameters $c_{L}$ and $c_{R}$, for which the last VEV was chosen to be $v_{N}=1 \mathrm{TeV}$. In the left panel, we see that the excited fermion corresponding to a left-handed zero mode localized towards the UV sites (corresponding to $c_{L}>0.5$ ) will have masses similar 
to the gauge bosons, i.e. or order $v_{N}$. However, for IR-localized left-handed zero modes $\left(c_{L}<0.5\right)$, the fermion excitation will become exponentially heavier. This is particularly so for low values of $N$, the number of sites in the quiver. On the other hand, the excited fermions corresponding to right-handed zero modes have the opposite behavior: there will be exponentially heavy when the zero mode is UV-localized, whereas they will be as light as the excited gauge bosons when the zero mode is localized towards the IR sites. This can be seen in the right panel of figure 4 . The reason for this differing behavior is rooted in the boundary conditions imposed to obtain a left- or right-handed zero modes.

The situation is very different from the continuum, where the excited fermions are lightest for $c_{L}=0.5$ or $c_{R}=-0.5$, and would become linearly heavier (as opposed to exponentially here) on both sides of these values. The behavior of the excited fermion masses with the localization parameters $c_{L}$ and $c_{R}$ will have important consequences phenomenologically. In general, we can say that the fermion excitations of left-handed zero modes localized in the IR (typically corresponding to heavier zero modes) will be considerably heavier than the gauge boson excitations, whereas the ones corresponding to UV-localization will be as light as them. The opposite will be the case for fermion excitations of right-handed zero modes: fermion excitations typically corresponding to the first two families will be heavier, whereas the excitation of the right-handed top quark should be as light as the gauge excitations. We will explore these important issues when we study the production and the decays of the quark excitations in section 5 .

We must note that this is not the only possible spectrum of fermion excitations, despite being the most common one. Depending on the details of the fermion representations in the quiver, it is possible to obtain important deviations from this behavior. For instance, it would be possible to obtain fermion resonances that are lighter than the gauge excitations. A similar situation is present in the continuum in the CHM [32]. More generally, it has been shown to be a generic occurrence even in discrete models [33]. The potential presence of light fermion excitations, particularly if associated to the top zero mode, is of great importance when addressing the tuning necessary to obtain a light Higgs. Thus, in order to have a natural Higgs sector we imagine that the full theory must have at least some of the fermion excitation to be rather light, particularly the top partners. The phenomenology associated with these light fermion excitations, has been studied in some detail in the continuum [34, 35], as well as in simplified versions of composite models [36]. Conversely, we can think of the model with the spectrum studied here as giving a fine-tuned Higgs sector, where the fine tuning is typically of the order of $v^{2} / v_{N}^{2}$. Then, if the scale of the new resonances is $O(1) \mathrm{TeV}$, this results in a fine-tuning of a few percent. As $v_{N}$ is increased the tuning worsens, but the model still solves the big hierarchy problem. So regardless of the issue of tuning, here we are interested in the phenomenology of the heavy fermion resonances in the spectrum.

Finally, it will be useful to have the wave functions of the excited states in the quiver, particularly to understand their couplings. Using the values for the localization parameters $c_{L}$ mentioned above, the wave-functions for the relevant fermion resonances are shown in figure 5, for two values of the number of sites: $N=4$ in the left panel, $N=15$ for the one on the right. For the right-handed zero-mode tower, using values of $c_{R}$ with the opposite sign of the ones used in figure 5 results in the same plots. 

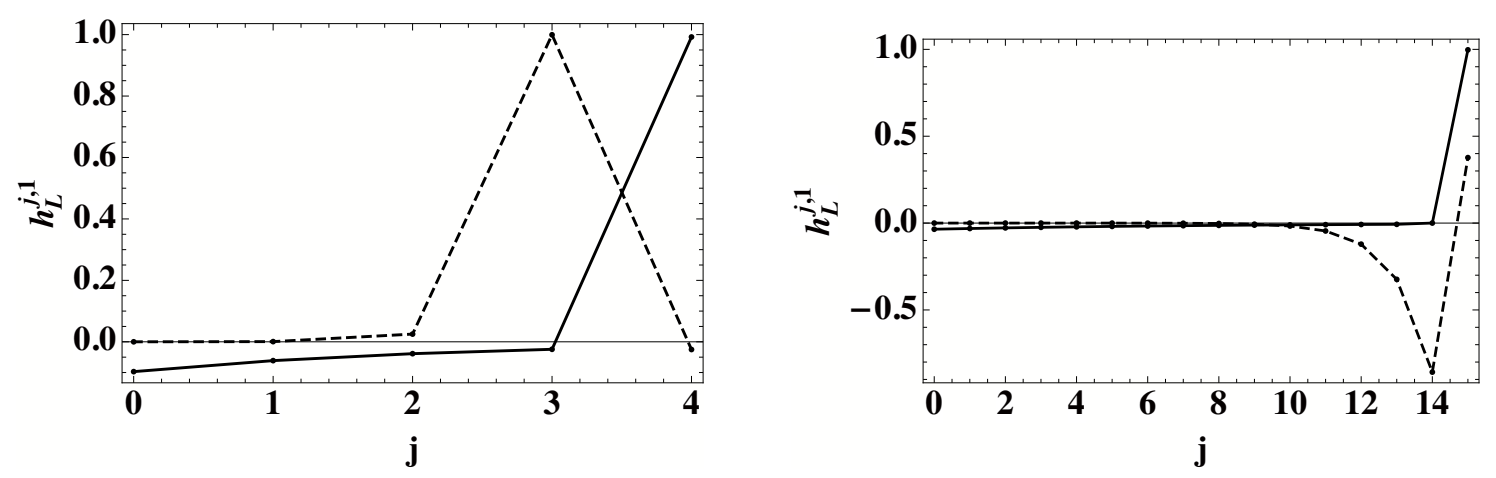

Figure 5. The wave-function of the first excitation of left-handed zero modes as a function of the site number $j$. Left panel: $N=4$, for $c_{L}=0.55$ (solid), $c_{L}=0.1$ (dashed). Right panel: $N=15$, for $c_{L}=0.55$ (solid), $c_{L}=0.1$ (dashed).

We can see that the excited fermions are generally IR localized, just as is the case in the continuum limit. However, the details of the wave function close to the IR can be important to determine the couplings to other states. For instance, depending on the value of the localization parameter, the wave function at the last site can be either large or rather suppressed. This will affect the couplings of the excited fermions to both the Higgs and the excited gauge bosons, which are important to determine the excited fermion decay channels. We will discuss this in detail in the next section.

\section{Couplings of the excited fermions}

We are interested in calculating the couplings of excited fermions in quiver theories which are relevant for their phenomenology. The production of these states will be dominated by the channels going through SM gauge bosons, as long as the excited fermion has SM quantum numbers. In particular, if we are interested in the largest production cross section, we will consider the excited quarks, which will be produced dominantly via QCD interactions, at least for moderate masses. On the other hand, when considering their decays, we will need to obtain the couplings of excited fermions to the zero modes and either excited gauge bosons or the Higgs doublet. This is due to the fact that decays require a non-diagonal coupling in the eigenstate quantum number $n$, and zero-mode gauge bosons do not allow it. The fact that both the excited gauge bosons and the Higgs have profiles that are IR localized allow for the occurrence of these couplings. The Higgs doublet couplings would result in a two-body decay of the excited fermions into the Higgs boson, as well as the longitudinal components of the $W$ and the $Z$. The decay channel induced by the nondiagonal couplings of the excited gauge bosons result in decays to them and a zero mode fermion too, but are phase-space suppressed by the proximity of the excited fermion and gauge boson masses. Although it then appears that the electroweak decays would dominate, under some circumstances the phase-space suppressed modes can be comparable or even dominate. This will depend on the details of the spectrum of excited fermions, which is highly flavor dependent as we saw in the previous section. 


\begin{tabular}{|c|c|cccc|}
\hline $\mathrm{N}$ & & $u^{(1)}$ & $d^{(1)}$ & $t^{(1)}$ & $b^{(1)}$ \\
\hline 4 & $L$ & 0.028 & 0.028 & 0.85 & 0.85 \\
& $R$ & $5 \times 10^{-4}$ & $1 \times 10^{-7}$ & 0.075 & 0.04 \\
\hline 15 & $L$ & 0.033 & 0.033 & 0.83 & 0.83 \\
& $R$ & $7 \times 10^{-4}$ & $2 \times 10^{-7}$ & 1.50 & 0.046 \\
\hline
\end{tabular}

Table 1. The couplings of the excited fermions to their zero mode and an excited gauge boson, in units of the zero-mode gauge couplings.

The couplings of excited fermions to a zero-mode fermion and an excited gauge boson can be computed by starting from the quiver gauge coupling

$$
\sum_{j=0}^{N} g_{j} \bar{\psi}_{L, R}^{j} \gamma^{\mu} \psi_{L, R}^{j} G_{\mu}^{j} .
$$

We can substitute for the rotation into mass eigen-states in Equation (4.1), and select the desired couplings. We obtain

$$
g_{L, R}^{101} \bar{\psi}_{L, R}^{(1)} \gamma^{\mu} \psi_{L, R}^{(0)} G_{\mu}^{(1)}+\text { h.c. }
$$

where we defined

$$
g_{L, R}^{101} \equiv \sum_{j=0}^{N} g_{j} h_{L, R}^{* j, 1} h_{L, R}^{j, 0} f^{j, 1},
$$

with $f^{j, 1}$ the wave function of the first excited state of the gauge boson as defined by the orthonormal rotation to the mass eigen-states

$$
G_{\mu}^{j}=\sum_{n=0}^{N} f^{j, n} G_{\mu}^{(n)} .
$$

Just as for the fermions, the $f^{j, n}$ 's are obtained by diagonalizing the gauge boson mass matrix $[18,19]$ that results in Equation $(2.1)$ when the link fields are written in terms of their VEVs as in Equation (2.2). Using the results for $f^{j, 1}$, as well as for the wave-functions of the zero mode and excited fermions, we can compute the couplings $g_{L, R}^{101}$ relevant for various cases. The results are summarized in table 1 . For instance, the first column corresponds to the couplings between the first-generation up excited quark $u^{(1)}$ to the zero mode, i.e the up quark, and a neutral excited gauge boson $G^{(1)}$ in units of the zero-mode gauge coupling, for left and right handed chiralities, and for $N=4$ or $N=15$. The largest couplings are found in the third generation, particularly the right-handed top sector. We will focus on the couplings to the excited gluon since they are the largest. However, if we were to consider a minimal theory without QCD propagating in the quiver diagram this would be an electroweak excited gauge boson.

The other important couplings of the excited fermions are those to the Higgs sector. These could dominate the excited fermion decays, particularly of the third generation, 
through channels like $f^{(1)} \rightarrow f^{(0)} \phi_{\alpha}$, where the $\phi_{\alpha}$ stands for the appropriate member of the Higgs doublet, i.e. either the Higgs boson, or the longitudinal components of the weak gauge bosons. To compute these couplings we take into account the general form of the fermion couplings to the link fields containing the Higgs doublet and given in Equation (3.1). The exact form of the couplings would generally depend on the details of the quiver theory: the gauge groups propagating and the chosen fermion representations. However, we would like to extract the generic behavior of these couplings by considering only the fermions with SM quantum numbers. This will be enough to obtain the couplings of the excited fermions to the Higgs doublet and the zero-mode fermions that will be present in all models, although it would ignore the potential contributions of exotic states which may arise in some specific realizations. We take this approach in order to be as model-independent as possible.

In general, there will be two types of Yukawa terms consistent with the quiver symmetries. The first type are those among the members of the same fermion tower: the sets of fermions $\psi_{L}^{j}$ and $\psi_{R}^{j}$ with a common zero mode These are the ones depicted in Equation (3.1) proportional to $\lambda$ and that can be obtained from the deconstruction of the $5 \mathrm{D}$ fermion kinetic term interacting with $A_{5}$. The second type, involves couplings between two different towers, such as

$$
\bar{\chi}_{R}^{j-1} \Phi_{j} \xi_{L}^{j}
$$

where $\xi_{L, R}^{j}$ corresponds to a tower with a zero mode different from that of $\chi_{L, R}^{j}$. This kind of coupling is gauge invariant and therefore allowed in the quiver theory, whereas it has no analog in the continuum limit. Finally, it is also possible to add terms at the $j=0$ and $j=N$ sites that are only invariant under the respective $\mathrm{SU}(2) \times \mathrm{U}(1)$ symmetries there. The couplings within each tower result in the mass matrices we diagonalized in the previous section and result in zero-mode masses and wave functions. With the additional contributions mentioned above, the breaking of the quiver symmetry down to $\mathrm{SU}(2) \times \mathrm{U}(1)$ leads to couplings of the Higgs doublet extracted from the pNGB surviving in the spectrum. The details of the resulting mixing spectrum are then heavily dependent on the specific model. However, it will always result in couplings that take the form

$$
\mathcal{L} \supset-\sum_{j=1}^{N} y_{j} \bar{\chi}_{R}^{j-1} b_{j} H \xi_{L}^{j}+\text { h.c. },
$$

where the Yukawa couplings $y_{j}$ are assumed to be $O(1)$, and the $\chi_{L, R}^{j}$ and $\xi_{L, R}^{J}$ are fermions propagating on the quiver with the appropriate quantum numbers and different zero modes. Here $H$ is the Higgs doublet

$$
H=\left(\begin{array}{c}
\phi^{+} \\
\frac{h+\phi^{0}}{\sqrt{2}}
\end{array}\right) .
$$

and the $b_{j}$ 's are defined in (2.12), so that $b_{j} H$ in (4.6) is the fraction of the pNGB Higgs at the site $j$.

We are interested in the couplings of the first fermion excitations to the Higgs doublet and a fermion zero mode. These arise due to the fact that both the excited fermion and 


\begin{tabular}{|c|c|c|c|c|c|c|c|}
\hline$N$ & $t_{R}^{(1)} t_{L}^{(0)}$ & $t_{L}^{(1)} t_{R}^{(0)}$ & $u_{R}^{(1)} u_{L}^{(0)}$ & $u_{L}^{(1)} u_{R}^{(0)}$ & $t_{L}^{(1)} b_{R}^{(0)}$ & $u_{L}^{(1)} d_{R}^{(0)}$ & $b_{R}^{(1)} t_{L}^{(0)}$ \\
\hline 4 & 0.365 & 0.028 & $4 \times 10^{-9}$ & 0.002 & 0.04 & $3 \times 10^{-6}$ & $2.6 \times 10^{-4}$ \\
15 & 0.18 & 0.35 & $3 \times 10^{-5}$ & $3 \times 10^{-4}$ & 0.014 & $1.3 \times 10^{-7}$ & 0.001 \\
\hline
\end{tabular}

Table 2. The couplings of the excited fermions to their zero mode and the Higgs doublet. From columns 3 to 5 , we show the couplings to the neutral Higgs sector, i.e. $h$ and $Z_{L}$. The last two columns correspond to the charged couplings to $W_{L}$.

the Higgs doublet have IR-localized wave functions different from the zero-mode fermion's. Making use of the rotation (3.2), we can express the Higgs doublet couplings in (4.6) as

$$
\mathcal{L} \supset-\left[\sum_{j=0}^{N} y_{j} h_{L}^{* j, 0} h_{R}^{j, 1} \frac{v_{N}}{v_{j}}\right] \bar{Q}_{L}^{(0)} H q_{R}^{(1)}+\text { h.c. }+\cdots,
$$

where we have extracted the couplings of interest, namely the one between the first quark excitation and a zero mode, which in this particular example gives the couplings of the first excitation of a right-handed zero-mode quark. In (4.8) the wave-functions $h_{L, R}^{j, 0}$ and $h_{L, R}^{j, 1}$ are the one we obtained in section 3 . In table 2 we show representative values of the couplings of excited fermions to the Higgs doublet and a zero mode for various quarks, for two cases $N=4$ and $N=15$. For instance, in the first column we have the coupling of the first excited right-handed top $t_{R}^{(1)}$ to the top quark and the neutral components of the Higgs doublet $H$.

The couplings vary a lot, mostly due to the changing zero-mode wave functions. These couplings allow us to compute the excited fermion decay into a zero mode and either the Higgs boson or the longitudinal components of the weak gauge bosons, $Z_{L}$ and $W_{L}^{ \pm}$. The last two columns are precisely the charged couplings for the third and first family excited fermions.

In the next section, we use the couplings computed here to study both the production and decay of the excited fermions.

\section{Phenomenology}

Here we consider the production and decay of the first excited quarks. The simplest mechanism is pair production via QCD. The alternative, is single production via electroweak boson exchange or gluon-weak boson fusion and can in principle be competitive for heavier masses. In figure 6 , the solid line shows the pair production of the first quark excitation $q^{(1)}$ via QCD. We ignored the negligibly small contributions mediated by the gauge excited states of the gluon. In this approximation, the curve is equally valid for all generations. The single production channel, through $W, Z$ exchange or $W, Z$ gluon fusion, requires electroweak couplings with a zero mode and the Higgs doublet. The relevant couplings are given in table 2 . We can see that they are only significant when considering the fermion excitations of the third-generation quarks. Even the production of $b_{R}^{(1)}$ is quite suppressed. On the other hand, single $b_{L}^{(1)}$ production through longitudinal $Z$ exchange can be significant. For illustrative purposes, here we consider the production of $t_{L, R}^{(1)}$, which is mediated by longitudinal $W$ exchange. With these couplings the single production of the top-quark 


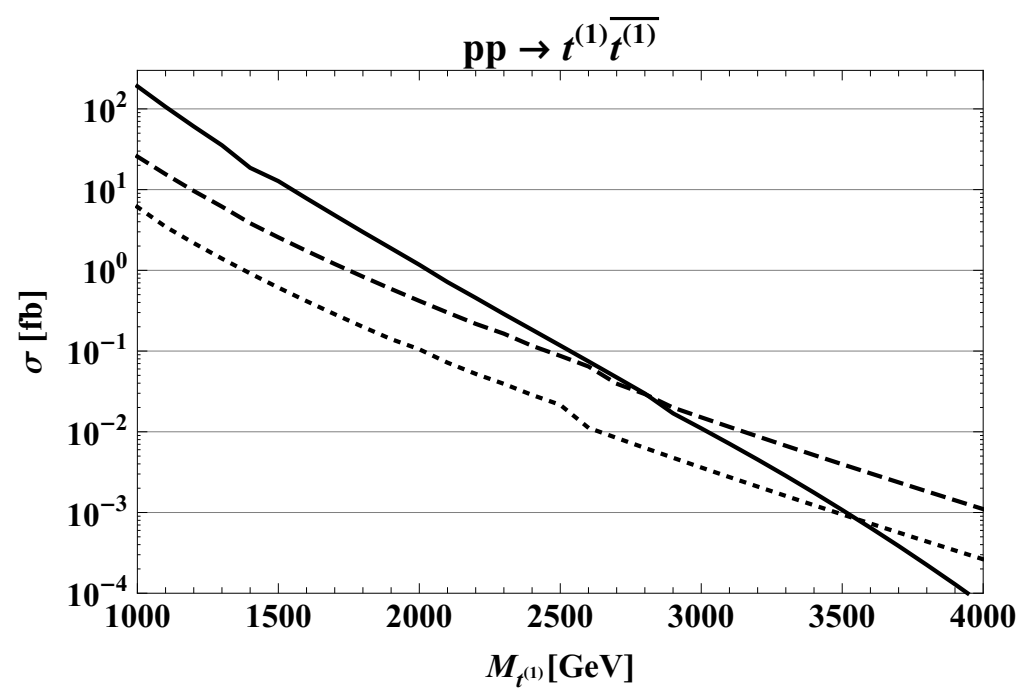

Figure 6. Cross sections for $t^{(1)}$ production, as a function of $M_{t^{(1)}}$ at $\sqrt{s}=14 \mathrm{TeV}$. The solid line is QCD pair production, whereas the dashed line corresponds to single production for $N=4$, and the dotted line to single production for $N=15$.
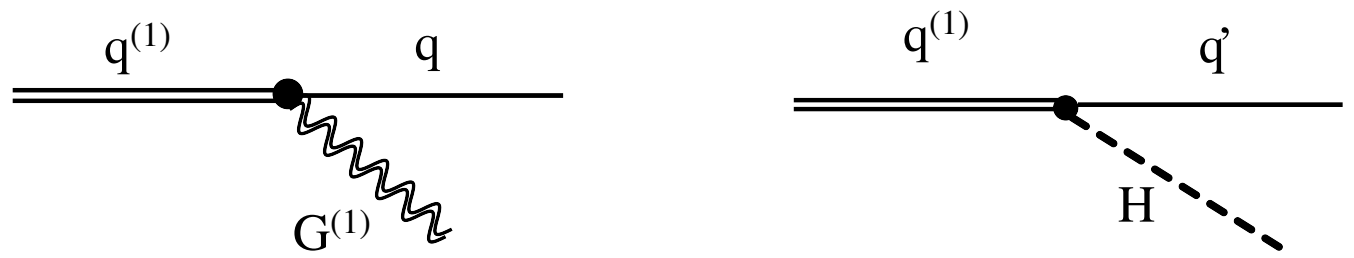

Figure 7. Decay modes for first excited quarks. The left diagram illustrates the decay into the heavy gluon and a zero mode through the couplings of table 1, whereas the right diagram shows the decay into electroweak states and a zero mode through the couplings of table 2.

excitation $t^{(1)}$, summing over the left and right-handed states, is given for $N=4$ and $N=15$ by the the dashed and dotted lines of figure 6 , respectively. In all cases we use the parton distribution functions from ref. [38]. We can see that pair production dominates over single production even for the cases with the largest couplings in table 2 , the top excited state. It is only for very large $t^{(1)}$ masses, $2.7 \mathrm{TeV}$ for $N=4$ and $3.5 \mathrm{TeV}$ for $N=15$, that the single production dominates due to the phase space suppression. But the production cross sections for these large masses are quite small. Then, for excited quark masses that will be accessible at the LHC in the next run, pair production dominates. The situation is very different than in other models with vector-like quarks, where the electroweak single production dominance appears at considerably smaller masses [37].

The production cross sections for pair production of all the excited quark states are the same for the same mass as long as the excited gluon contribution is neglected. However there are differences both in their spectrum and the couplings responsible for their decays.

There are two main mechanisms for the decay of the excited quarks, illustrated in figure 7. The electroweak-mediated two-body decays into the Higgs or the longitudinal components of the gauge bosons arise through the couplings shown in table 2 . There is 
also the decay into a gauge excitation, dominantly the heavy gluon, and the corresponding zero-mode quark, where the relevant couplings are the ones in table 1 . The latter mode is suppressed by phase space given that the mass difference between the quark and gauge excitations can be very small for certain values of the localization parameters, as illustrated in figure 4. However, the excited gauge boson mode can be competitive in many cases given that sometimes it only takes a small mass difference for it to have a branching fraction comparable to the electroweak modes. From figure 4 we see that only of $c_{L}>0.55$ and $c_{R}>-0.55$, the mass degeneracy between the fermion and the gauge boson excitations is close enough to suppress this mode.

Based on the above discussion, we see that the decay of $t_{R}^{(1)}$, the excited, vector-like fermion belonging to the tower with the right-handed top as its zero mode, is dominated by the electroweak modes since all typical solutions for the masses and mixings require this zero mode to be IR-localized, i.e. $c_{R}^{3}>-0.5$. Thus, ignoring the highly suppressed excited gauge boson mode, the branching ratios for $t_{R}^{(1)}$ decay should be

$$
\operatorname{Br}\left(t_{R}^{(1)} \rightarrow t h\right) \simeq \operatorname{Br}\left(t_{R}^{(1)} \rightarrow t Z_{L}\right) \simeq \frac{1}{2} \operatorname{Br}\left(t_{R}^{(1)} \rightarrow b W_{L}\right) .
$$

For the left-handed third-generation excitations $t_{L}^{(1)}$ and $b_{L}^{(1)}$, the situation is more model-dependent. For instance, for $N=4$ the electroweak coupling leading to the decays into weak bosons or the Higgs is somewhat suppressed (see table 2), while the decay to a zero mode and an excited gluon is not (table 1 ). The ratio of the two partial widths is

$$
\frac{\Gamma_{G}}{\Gamma_{H}}=\frac{8}{3}\left(\frac{g_{G}^{101}}{g_{H}^{10}}\right)^{2} \frac{\left(M_{1}^{2}-M_{G}^{2}\right)^{2}}{M_{1}^{4}},
$$

where $M_{G}$ is the mass of the first gluon excitation, and $g_{G}^{101}$ and $g_{H}^{10}$ are the coupling of $t^{(1)}$ to its zero mode and an excited gluon and to the Higgs doublet and a zero mode, respectively. We can now estimate then that in order for these channels to be comparable in this case the mass difference $\Delta M=M_{1}-M_{G}$ needs to satisfy

$$
\frac{\Delta M}{M_{1}}>0.02
$$

The solutions for the localization parameters we have used so far are such that $c_{L}^{3}=0.55$, which by inspecting figure 4 appears to give values of $\Delta M$ just a bit smaller than the bound on (5.3). But it is clear that for values only slightly smaller of $c_{L}^{3}$ we would already satisfy this condition. Since obtaining mass and CKM solutions with these values of $c_{L}^{3}$ is perfectly feasible, we conclude that both decay modes are likely and must be considered.

The situation is similar for the left-handed quark excitations of the first and second families. For instance, for the first family, we see from tables 1 and 2 that the $u_{L}^{(1)}$ couplings to the Higgs doublet and a zero mode are quite suppressed compared to the non-diagonal excited gluon couplings. Even when considering the fact that $c_{L}^{1}>0.55$ is always satisfied for most solutions (i.e. first-generation zero mode quarks must be UV-localized), very small values of $\Delta M$ are required in order for the excited gluon mode to be important. E.g. for $N=4$ we need $\Delta M / M_{1}>0.02$, whereas for $N=15$ just having $\Delta M / M_{1}>0.005$ is 
enough. For the second-generation quark excitations this is even more common, given the slightly smaller values of $c_{L}^{2}$ which allow for larger masses for them (see figure 4).

Finally, we consider the decays of the excitations of the first and second generation right-handed quarks. Let us focus on $u_{R}^{(1)}$, but similar conclusions will apply to $d_{R}^{(1)}$ as well as to the analogous second generation excitations. From table 2 we can see that the couplings to $H$ and a zero mode that govern the electroweak decay mode are highly suppressed, both for low $(N=4)$ and moderate $(N=15)$ number of sites, relative to the couplings to the gluon excitation (table 1 ). Thus mass differences only need to be very small $\left(\Delta M / M_{1}>2 \times 10^{-4}\right.$ for $N=4, \Delta M / M_{1}>0.01$ for $\left.N=15\right)$, which is almost always satisfied in most cases. Thus, it is very likely that these excited quarks decay exclusively through the heavy gluon decay mode. A very similar situation occurs with $b_{R}^{(1)}$.

To summarize, the decays of $t_{R}^{(1)}$ are likely to be dominated by the electroweak channels: $t_{R}^{(1)} \rightarrow\left(h, Z_{L}\right) t$ and $t_{R}^{(1)} \rightarrow b W_{L}$. On the other hand, the decays of all other right-handed excitations $\left(b_{R}^{(1)}, u_{R}^{(1)}\right.$, etc.) are most likely dominated by the heavy gluon mode, as in $u_{R}^{(1)} \rightarrow u G$. Finally, the left-handed excitations $\left(t_{L}^{(1)}, u_{L}^{(1)}\right.$, etc.) have couplings leaving in the boundary between the dominance of the two channels, and in general is possible that both decay channels are present.

Bounds on the masses of the excited quarks, mainly those corresponding to third generation zero modes, are obtained at the LHC by ATLAS and CMS through the various electroweak decay modes [39-42]. From the latest analyses of the $\sqrt{s}=8 \mathrm{TeV}$ data the bounds imply that the masses must be typically below $600 \mathrm{GeV}$ to almost $800 \mathrm{GeV}$, depending on the assumptions regarding branching fractions. For instance for $t_{R}^{(1)}$, given the electroweak branching ratio dominance discussed above, we can deduce a bound of about $M_{1}>696 \mathrm{GeV}$ from refs. [40-42]. In any case, the current bounds are all below $1 \mathrm{TeV}$. On the other hand, for the heavy gluon decay modes, $q^{(1)} \rightarrow q G^{(1)}$, the final states are: $t \bar{t}+$ hard jet, $b \bar{b}+$ hard jet or simply hard jets. As a final comment, we should have in mind that is possible to build quiver theories of EWSB and fermion masses without the gluon excitations. Thus, the decay mode into heavy gauge bosons, competing with the transitions into the Higgs sector, are those to the excitations of the $W$, the $Z$ and the photon. More detailed studies of all these final states, as well as of the electroweak decay modes for heavier masses, are left for future work [43].

\section{Conclusions and outlook}

Quiver theories with a pNGB Higgs are a natural extension of the SM. Although they are closely related to holographic/ $\mathrm{AdS}_{5}$ models, they correspond to their coarse deconstruction and have significant quantitative differences with them. In this paper we studied the fermion excitations in these models by obtaining their spectrum, couplings to excited gauge bosons and the Higgs sector, and the resulting phenomenology in their production and decays. We have focused on the quark excitations, and in additions we choose the minimum quark content in the quiver that has to be present to reproduce the SM quark sector. Thus, the quark excitations studied in this paper are just the ones with a SM counterpart. The advantage is that their properties are largely model independent, and in particular do 
not depend on the fermion representation in the quiver. The obvious drawback of this general approach is that the spectrum of quark excitations is incomplete. In particular, with this spectrum of excitations alone the Higgs sector will require some tuning in order to result in a Higgs mass as light as the one observed. One could imagine that, just as it is the case in continuum models, extending the fermion representation might result in lighter fermions which would ameliorate this problem. Conversely, we could accept this fine-tuning, already of the order of a few percent or larger, given that the model solves the big hierarchy problem. Either way, the phenomenolgy of the heavy spectrum of fermion resonances needs to be studied.

An important difference with the continuum $\mathrm{AdS}_{5}$ models and the holographic realizations inspired by them can already be seen in the spectrum of fermion excitations, obtained in section 3. This can be appreciated in both panels of figure 4, which show the dependence of the fermion excitation mass $M_{1}$ with the localization parameters for left and right handed zero modes. For instance, for the excitation with a left-handed zero mode we see that $M_{1}$ saturates towards the mass of the gauge excitation $(1 \mathrm{TeV}$ in the example of figure 4) for values of the localization parameter $c_{L}$ corresponding to UV localization, whereas for smaller values corresponding to IR localization $M_{1}$ growths exponentially with respect to the gauge excitation mass. On the other hand, for the excitation with a righthanded zero mode the behavior is the opposite: $M_{1}$ saturates towards the gauge excitation mass for values of $c_{R}$ consistent with IR localization of the zero mode. This is in stark contrast with the continuum, which exhibits in both cases a behavior symmetrical with respect to $c_{L}=0.5$ and $c_{R}=-0.5$, with $M_{1}$ growing linearly from these points. We have shown in section 4 and particularly in section 5 that this has important consequences in the phenomenology of the production and decay of these fermion excitations. Namely the special pattern of decays of the various quark excitations is in great part determined by this feature. We see one more time, just as it was the case for the gauge excitations studied in [22], that there are important phenomenological differences between quiver theories and their continuum cousins. In this case, they point to a fundamental aspect of the theory determining the spectrum of fermion resonances.

The examples studied here are obtained for a specific solution of the localization parameters $c_{L, R}$ compatible with the SM quark masses and CKM mixing [20]. Although other solutions might be possible, we believe that the general features found here should persist. A more detailed study of the phenomenology of the quark excitations at the LHC, including backgrounds and search strategies, is left for future work [43]. Similarly, the study of the lepton excitations will be done separately since it requires the input of the lepton sector of the SM, which involves not only the spectrum of neutrinos with the need of a see-saw mechanism, but also their particular mixing [24].

\section{Acknowledgments}

The authors acknowledge the support of the State of São Paulo Research Foundation (FAPESP), and the Brazilian National Council for Technological and Scientific Develop- 
ment $(\mathrm{CNPq})$. V. P. acknowledges the support of the Committee for the Advancement of Higher Education (CAPES).

Open Access. This article is distributed under the terms of the Creative Commons Attribution License (CC-BY 4.0), which permits any use, distribution and reproduction in any medium, provided the original author(s) and source are credited.

\section{References}

[1] ATLAS collaboration, Observation of a new particle in the search for the standard model Higgs boson with the ATLAS detector at the LHC, Phys. Lett. B 716 (2012) 1 [arXiv: 1207.7214] [INSPIRE].

[2] CMS collaboration, Observation of a new boson at a mass of $125 \mathrm{GeV}$ with the CMS experiment at the LHC, Phys. Lett. B 716 (2012) 30 [arXiv:1207.7235] [INSPIRE].

[3] H. Baer and X. Tata, Weak scale supersymmetry: from superfields to scattering events, Cambridge Univ. Pr., Cambridge U.K. (2006) [INSPIRE].

[4] N. Arkani-Hamed, A.G. Cohen and H. Georgi, Electroweak symmetry breaking from dimensional deconstruction, Phys. Lett. B 513 (2001) 232 [hep-ph/0105239] [INSPIRE].

[5] N. Arkani-Hamed, A.G. Cohen, E. Katz, A.E. Nelson, T. Gregoire et al., The minimal moose for a little Higgs, JHEP 08 (2002) 021 [hep-ph/0206020] [INSPIRE].

[6] D.E. Kaplan and M. Schmaltz, The little Higgs from a simple group, JHEP 10 (2003) 039 [hep-ph/0302049] [INSPIRE].

[7] For a review see M. Schmaltz and D. Tucker-Smith, Little Higgs review, Ann. Rev. Nucl. Part. Sci. 55 (2005) 229 [hep-ph/0502182] [INSPIRE].

[8] R. Contino, Y. Nomura and A. Pomarol, Higgs as a holographic pseudo-Goldstone boson, Nucl. Phys. B 671 (2003) 148 [hep-ph/0306259] [inSPIRE].

[9] K. Agashe, R. Contino and A. Pomarol, The minimal composite Higgs model, Nucl. Phys. B 719 (2005) 165 [hep-ph/0412089] [INSPIRE].

[10] D. Marzocca, M. Serone and J. Shu, General composite Higgs models, JHEP 08 (2012) 013 [arXiv: 1205.0770] [INSPIRE].

[11] L. Randall and R. Sundrum, A large mass hierarchy from a small extra dimension, Phys. Rev. Lett. 83 (1999) 3370 [hep-ph/9905221] [INSPIRE].

[12] K. Agashe, A. Delgado, M.J. May and R. Sundrum, RS1, custodial isospin and precision tests, JHEP 08 (2003) 050 [hep-ph/0308036] [INSPIRE].

[13] G. Burdman, Constraints on the bulk standard model in the Randall-Sundrum scenario, Phys. Rev. D 66 (2002) 076003 [hep-ph/0205329] [INSPIRE].

[14] G. Burdman and Y. Nomura, Holographic theories of electroweak symmetry breaking without a Higgs boson, Phys. Rev. D 69 (2004) 115013 [hep-ph/0312247] [InSPIRE].

[15] C. Csáki, A. Falkowski and A. Weiler, The flavor of the composite pseudo-Goldstone Higgs, JHEP 09 (2008) 008 [arXiv:0804.1954] [INSPIRE].

[16] N. Arkani-Hamed, A.G. Cohen and H. Georgi, (De)constructing dimensions, Phys. Rev. Lett. 86 (2001) 4757 [hep-th/0104005] [INSPIRE]. 
[17] C.T. Hill, S. Pokorski and J. Wang, Gauge invariant effective Lagrangian for Kaluza-Klein modes, Phys. Rev. D 64 (2001) 105005 [hep-th/0104035] [INSPIRE].

[18] A. Falkowski and H.D. Kim, Running of gauge couplings in AdS $S_{5}$ via deconstruction, JHEP 08 (2002) 052 [hep-ph/0208058] [INSPIRE].

[19] L. Randall, Y. Shadmi and N. Weiner, Deconstruction and gauge theories in AdS $S_{5}, J H E P 01$ (2003) 055 [hep-th/0208120] [INSPIRE].

[20] G. Burdman, N. Fonseca and L. de Lima, Full-hierarchy quiver theories of electroweak symmetry breaking and Fermion masses, JHEP 01 (2013) 094 [arXiv: 1210.5568] [INSPIRE].

[21] Y. Bai, G. Burdman and C.T. Hill, Topological interactions in warped extra dimensions, JHEP 02 (2010) 049 [arXiv:0911.1358] [INSPIRE].

[22] G. Burdman, N. Fonseca and G. Lichtenstein, Resonances from quiver theories at the LHC, Phys. Rev. D 88 (2013) 116006 [arXiv:1308.5988] [INSPIRE].

[23] G. Burdman, N. Fonseca and L. Lima, The pNGB Higgs in quiver theories, in preparation.

[24] G. Burdman, L. Lima, R. D'Elia Matheus and C. Machado, Leptons in quiver theories with a $p N G B$ Higgs, in progress.

[25] R. Contino, T. Kramer, M. Son and R. Sundrum, Warped/composite phenomenology simplified, JHEP 05 (2007) 074 [hep-ph/0612180] [INSPIRE].

[26] S. De Curtis, M. Redi and A. Tesi, The 4D composite Higgs, JHEP 04 (2012) 042 [arXiv:1110.1613] [INSPIRE].

[27] R.S. Chivukula, B. Coleppa, S. Di Chiara, E.H. Simmons, H.-J. He et al., A three site Higgsless model, Phys. Rev. D 74 (2006) 075011 [hep-ph/0607124] [INSPIRE].

[28] G. Panico and A. Wulzer, The discrete composite Higgs model, JHEP 09 (2011) 135 [arXiv:1106.2719] [INSPIRE].

[29] H. Abe, T. Kobayashi, N. Maru and K. Yoshioka, Field localization in warped gauge theories, Phys. Rev. D 67 (2003) 045019 [hep-ph/0205344] [INSPIRE].

[30] J. de Blas, A. Falkowski, M. Pérez-Victoria and S. Pokorski, Tools for deconstructing gauge theories in $A d S_{5}$, JHEP 08 (2006) 061 [hep-th/0605150] [INSPIRE].

[31] T. Gherghetta and A. Pomarol, Bulk fields and supersymmetry in a slice of AdS, Nucl. Phys. B 586 (2000) 141 [hep-ph/0003129] [INSPIRE].

[32] R. Contino, L. Da Rold and A. Pomarol, Light custodians in natural composite Higgs models, Phys. Rev. D 75 (2007) 055014 [hep-ph/0612048] [INSPIRE].

[33] O. Matsedonskyi, G. Panico and A. Wulzer, Light top partners for a light composite Higgs, JHEP 01 (2013) 164 [arXiv: 1204.6333] [INSPIRE].

[34] R. Contino and G. Servant, Discovering the top partners at the LHC using same-sign dilepton final states, JHEP 06 (2008) 026 [arXiv:0801.1679] [INSPIRE].

[35] B. Gripaios, T. Müller, M.A. Parker and D. Sutherland, Search strategies for top partners in composite Higgs models, JHEP 08 (2014) 171 [arXiv: 1406.5957] [INSPIRE].

[36] A. De Simone, O. Matsedonskyi, R. Rattazzi and A. Wulzer, A first top partner hunter's guide, JHEP 04 (2013) 004 [arXiv: 1211.5663] [INSPIRE].

[37] T. Han, H.E. Logan, B. McElrath and L.-T. Wang, Phenomenology of the little Higgs model, Phys. Rev. D 67 (2003) 095004 [hep-ph/0301040] [INSPIRE]. 
[38] A.D. Martin, W.J. Stirling, R.S. Thorne and G. Watt, Parton distributions for the LHC, Eur. Phys. J. C 63 (2009) 189 [arXiv:0901.0002] [inSPIRE].

[39] ATLAS collaboration, Search for pair and single production of new heavy quarks that decay to a $Z$ boson and a third generation quark in pp collisions at $\sqrt{s}=8$ TeV with the ATLAS detector, ATLAS-CONF-2014-036, CERN, Geneva Switzerland (2014).

[40] CMS collaboration, Inclusive search for a vector-like $T$ quark with charge $2 / 3$ in pp collisions at $\sqrt{s}=8 \mathrm{TeV}$, Phys. Lett. B 729 (2014) 149 [arXiv:1311.7667] [InSPIRE].

[41] CMS collaboration, Search for vector-like $b^{\prime}$ pair production with multilepton final states in $p p$ collisions at $\sqrt{s}=8 \mathrm{TeV}$, CMS-PAS-B2G-13-003, CERN, Geneva Switzerland (2013).

[42] CMS collaboration, Search for pair-produced vector-like quarks of charge $-1 / 3$ in dilepton+jets final state in pp collisions at $\sqrt{s}=8 \mathrm{TeV}$, CMS-PAS-B2G-12-021, CERN, Geneva Switzerland (2012).

[43] G. Burdman, P. Ormonde, B. Panes and V. Peralta, Signals for excited quarks from quiver theories at the $L H C$, in progress. 